\title{
Physiological responses over CrossFit athletes in the Brazil CrossFit championship
}

\author{
Zecchin-Oliveira AM" ${ }^{1,4 *}$, Dechechi $\mathrm{CJ}^{1,4}$, Menezes-Reis $\mathrm{R}^{2}$, Ribeiro VMB ${ }^{3}$, Fujita $\mathrm{RA}^{4,5}$ and Puggina EF ${ }^{1,4}$ \\ ${ }^{1}$ Ribeirao Preto Medical School (FMRP), USP-RP, Brazil \\ ${ }^{2}$ Human Talent School (FACTHUS), Uberaba, Brazil \\ ${ }^{3}$ Federal Institute of São Paulo, Brazil \\ ${ }^{4}$ School of Physical Education and Sport of Ribeirao Preto (EEFERP), USP-RP, Brazil \\ ${ }^{5}$ Ribeirão Preto College of Nursing (EERP), USP-RP, Brazil
}

\begin{abstract}
CrossFit is a high intensity interval training program. Is was founded in 2000, becoming a very common practice in many countries. CrossFit can improve general resistance condition enabling numerous benefits. Well trained CrossFit practitioners seen to take lower intensity than elite CrossFit athletes, and it leads researchers to believe that CrossFit is a fast adaptation program through different group of age and levels. The main objective of the present study was to evaluate 5 elite CrossFit athletes analysing real time load physiological internal load in the semi-final of Brazil CrossFit Championship.

Athletes showed great changes in all physiological measures after semi-final. There was physiological difference between athletes. The present study concluded that elite CrossFit athletes can withstand heavy training loads with great physiological changes, supporting the idea of fatigue/overtraining and recovery necessity in CrossFit competition. There is great difference demand in physiological values between elite athlete levels, expending more energy athletes who shows bigger heart rate.
\end{abstract}

\section{Introduction}

Founded in 2000, CrossFit is a high intensity interval training program that can improve physiological responses enabling numerous benefits [1-3]. CrossFit became world known, due it's high functionality, englobing high intensity exercise made in constantly variated form; exercises such as metabolic conditioning (running, bike, row, skip rope, swimming, skiing, etc.), Olympic Gymnastic (ring dip, ring row, hand stand walk, hand stand push-ups, etc.) and weightlifting (deadlift, back squat, snatch, clean, jerk, etc.) [4,5]. Since 2013, the number of affiliates more than doubled, from 5000 CrossFit centre (box) to 13.000 , with more than 300.000 practitioners around the world and it encourages sports scientists to research CrossFit [6]. Physiological responses in CrossFit Athletes is poorly studied. Most of the CrossFit studies evaluated injury and physical capacity, in eventual practitioners (none in elite athletes). The basic physiological responses such intensity analyses of the competition, excessive postexercise oxygen consumption (EPOC) levels and calories expenditure are remaining unknown in elite CrossFit athletes. Recently studies evaluating elite CrossFit athletes exposed activation of aerobic and anaerobic responses through short and very intense workout of the day (WOD [specific CrossFit training]) and high lactate concentration after one WOD englobing moderate volume and high intensity $[7,8]$. A recent study evaluates physiological levels of testosterone, IL-1, IL10, etc in CrossFit amateur athletes in a competition. After the last day of the competition, testosterone and IL-10 (anti-inflammatory interleukin) levels were diminished, demonstrating hormonal and molecular disturbance, giving the idea of the importance of life style to be able to wellbeing in competition and adequate periodization to participate of a competition [9]. Well trained CrossFit practitioners seem to take lower intensity than elite CrossFit athletes, and it leads researchers to believe that CrossFit is a fast adaptation practice through different group of age and levels [8-11]. Through the stress given by CrossFit, physiological factors tend to exacerbate. Schubert at al. found that amateur CrossFit practitioners can account for a significant portion of daily energy expenditure, and consecutive training can induce meaningful weight loss [12].

Elite athletes can worse their competition development because of many factors, such as physiological (expending calories cited above, excessive intensity, overtraining due past trainings, etc.) and psychological (internal or external stress, family support, etc.) $[13,14]$.

In this paper, detailed physiological characteristics of the CrossFit elite athletes over a competition that leads to the world CrossFit championship (CrossFit Games).

To the best of our knowledge this is the first study analysing physiological responses in performance during a competition, that leads to the CrossFit Games in elite athletes during an official competition. In this paper detailed characteristics of the physiological internal load in real time in the semi-final of Brazil CrossFit Championship (BCC).

\section{Methods}

4 elite men and 1 elite woman CrossFit Athletes (2 males for individual (elite athlete $\mathrm{A}$ and elite athlete $\mathrm{B}$, (EAA and EAB)) competition and 2 males and 1 female (EAC) for team competition

${ }^{\star}$ Correspondence to: Zecchin-Oliveira AM, Ribeirao Preto Medical School (FMRP), USP-RP, Brazil, E-mail: arthurzecchin@usp.br

Key words: HIIT, extreme training, Cross training, competition, performance

Received: February 20, 2020; Accepted: March 17, 2020; Published: March 20, 2020 
were analysed in the 2019 semi-final of BCC, that is one of the phases of competition which lead the winners to the CrossFit Games; each phase there is only one man and 1 woman, and 1 team (the $1^{\circ}$ place of each category) that is able to compete the CrossFit Games. To participate in the BCC there is a qualifier which the best 28 individual athletes and 14 team athletes are classified.

The analysed semi-final WOD in individual group was: "Up and side down" for time. All the athletes must perform in 7 minutes, 10-86-4-2 repetitions of muscle-ups (very common movement in Olympic gymnastics composed by holding the suspended rings and throwing the body over the rings with specific technique) and 16 meters of hand stand walk (walk using hands in upside down position) after each round.

The analysed semi-final WOD in team was: "The long way home" for time: All the teams must perform 5 rounds for time (12'). The team must complete 10 ring muscle-ups (one athlete do the movements and the others athletes must hold the anaconda $(176 \mathrm{~kg})-10$ anaconda thrusters (all the team together).

The main instrument used to analyse the physiological responses in the selected elite athletes was the Firstbeat Sports ${ }^{\otimes}$ (Firstbeat Sport, Finland), and it was exported to the Firstbeat Sports Server software (version 4.7.3.1). The physiological responses analysed was calories (kcal), medium heart rate (MHR), training load in arbitrary units ((AU)TRIMP), Excessive post-exercise oxygen consumption (EPOC) and training effect. Training effect predicts how the effort will impact the $\mathrm{VO}_{2}$ max fitness level through the EPOC (Figure 1). The rate perceived exertion (RPE) was analysed, it was originally proposed by Borg, and modified by Foster et al. $[15,16]$ using time of the exercise performed $x$ RPE. RPE was measured 30 minutes after the WOD with the athletes pointing 0 to 10 foster scale (from $0=$ rest to $10=$ extremely intense), measuring the intensity of the WOD. 1 repetition maximum (1RM) of back squat propose by Kraemer et al. [17] and the "FRAN" WOD was used to determine strength and intensity [18]. The WOD named "FRAN" is one of the most common WODS, it was used to describe individual performance condition. The FRAN WOD is composed by 21, 15 and 9 repetition of thruster exercise (the barbell starts in shoulder position and the athlete must do a front squat with the extension of elbows at the end of hip extension) and pull-up (the athlete must hang the fixed bar and start from stretched elbows to bend elbows until the chin goes up to the bar), as fast as possible (for time). This WOD was described before [19,20].

Prior to the case report, the participant signed a written informed consent, which was approved by the local ethics committee (Protocol number: 13353719.4.0000.5659).

Statistical analyses: It wasn't made because the participants number is too low. So, this is only an observational study.

\section{Results}

The characteristics of each athlete are exposed in table 1.

EAA did not do the CrossFit Open due to a knee injury (he was classified to the BCC qualifier before the injury). Only the EAC was classified to the BCC finals, but she didn't classify to the CrossFit Games.

Table 2 exposes the physiological values obtained in real-time assessment during the semi-final WOD.

Training Effect showed improvement in $\mathrm{VO}_{2}$ max in both athletes (Table 2 and Figure 1).
Table 1a. Elite athletes characteristic (individual competition)

\begin{tabular}{|l|c|c|}
\hline & EAA & EAB \\
\hline Age & 30 & 23 \\
\hline Weight $(\mathrm{kg})$ & 80 & 90 \\
\hline BMI & 25,88 & 26,62 \\
\hline 1RM Back Squat (kg) & 170 & 195 \\
\hline WOD “Fran” best time & $28^{\prime}$ ” & $22^{\prime}$ \\
\hline 2019 CrossFit Open ranking (Brazil) & - & $12^{\circ}$ \\
\hline 2019 CrossFit BCC semifinal & $26^{\circ}$ & $26^{\circ *}$ \\
\hline Final Position BCC & $29^{\circ}$ & $15^{\circ}$ \\
\hline
\end{tabular}

*Draw event

Table 1b. Elite athletes characteristic (team competition)

\begin{tabular}{|l|c|c|c|}
\hline & EAC* & EAD & EAE \\
\hline Age & 28 & 24 & 21 \\
\hline Weight (kg) & 64 & 92 & 96 \\
\hline BMI & 27.35 & 28.39 & 27.82 \\
\hline 1RM Back Squat (kg) & 145 & 211 & 205 \\
\hline WOD “Fran" best time & $22^{\prime} 28^{\prime}$ & $2^{\prime} 32^{\prime \prime}$ & $2^{\prime} 15^{\prime}$ \\
\hline 2019 CrossFit Open ranking (Brazil) & $1^{\circ}$ & $5^{\circ}$ & $5^{\circ *}$ \\
\hline 2019 CrossFit BCC semifinal & $4^{\circ}$ & $17^{\circ}$ & $13^{\circ}$ \\
\hline Final Position BCC & $4^{\circ}$ & $11^{\circ}$ & $13^{\circ}$ \\
\hline
\end{tabular}

** Female athlete; *Draw competition

Table 2. Physiological characteristics

\begin{tabular}{|l|c|c|c|c|c|}
\hline & EAA & EAB & EAC & EAD & EAE \\
\hline Cal & 109 & 74 & 94 & 116 & 186 \\
\hline MHR & 168 & 170 & 167 & 166 & 174 \\
\hline Training load (AU) & 106 & 27 & 26 & 24 & 38 \\
\hline EPOC & 112 & 66 & 61 & 55 & 84 \\
\hline RPE & 9 & 8 & 7 & 7 & 8 \\
\hline Training Effect & 3.3 & 3.1 & 2.3 & 2.2 & 3.4 \\
\hline
\end{tabular}

* Cal: calories (Kcal); MHR: medium rate rate (BPM); TRIMP: training impulse; EPOC: excessive post exercise oxygen consumption; RPE: Rating perceived exertion; Perceived Training effect: based on VO2max fitness level.

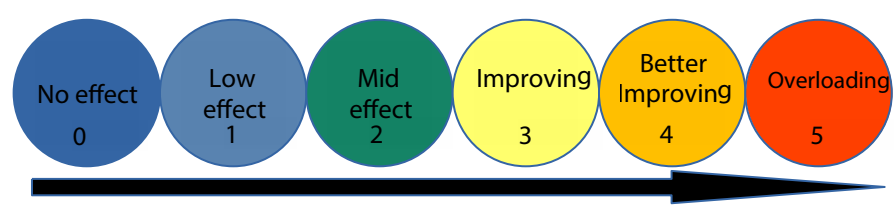

TIME

Figure 1. Training effect levels

\section{Discussion}

The present study is a new way of investigation to the coaches understand how the athlete physiological responses over a competition in real time mensuration is. The purpose of the present study was about tracking physiological responses (performance) in elite CrossFit athletes in real time over one considerable CrossFit championship. Furthermore, the present study exposed the impact performance of freshly treated injury in elite athlete in one international competition.

Our results showed high RPE in an individual athlete (EAA) when compared with $\mathrm{EAB}$ and team athletes, but all the athletes demonstrated high RPE. Because this WOD was made in one semi-final of a CrossFit Games stage, these athletes gave the best to classify (increasing intensity to almost $100 \%$ of RPE). These results confront the study of Shaw et al. 2015 who found moderate intensity in well-trained practitioners in a single bout of CrossFit WOD, perhaps because it wasn't an important competition [21]. A recent study evaluated two CrossFit workouts and the intensity. One with high WOD volume and one with low WOD 
volume. The results showed that low WOD volume exhibits lower RPE than high volume, but lactate was higher in the lower WOD volume, concluding that higher WOD volume can increase more intensity through lactate, but not through RPE. The semi-final WOD can be classified as low WOD volume (lower than 10') in individuals and medium volume (more than 10') for the teams.

The WOD "FRAN" which is performance reference in CrossFit practitioners showed better WOD time in EAA than EAB, and it had been previously demonstrate high correlation with back squat 1RM [4], confirmed in the present study (EAA with bigger RM back squat than EAB). EAE showed better "FRAN" time than the others team athletes and individual athletes (EAD and EAE showed back squat RM higher than $200 \mathrm{~kg}$ ).

The individual WOD had a duration of 7 minutes and none of these two athletes completed the WOD in the time cap (limit time). The kcal spent in the short WOD was higher in EAA. EAE demonstrated higher energy expenditure when compared with individual and team athletes. Interestingly there is relationship between MHR and Cal, as bigger is the MHR, bigger is the energy expenditure. According to the intensity of exercise increases, the HR and the total calories increase faster. A study analysing the effect of caffeine (caffeine vs placebo) on energy expenditure showed that hypertrophy training at a gym at sub maximal intensity until failure (bout of 45-50 minutes at 70-80\%1RM) expended medium of $137 \mathrm{kcal}$ (caffeine) and $118 \mathrm{kcal}$ (placebo) after 75 minutes past High volume training at submaximal intensity have similar kcal expenditure that 7 minutes high intensity interval training [22].

Some studies investigated the intensity of CrossFit training in injury risks, correlating higher intensity and fatigue with higher levels of injury $[23,24]$. The present study could analyse the training effect level (exposed in Figure 1) of these elite athletes, and it was demonstrated that the intensity was enough to improve aerobic capacity and not overloading it. It is believed that the semi-final of CrossFit BCC didn't increase the level of all athletes to be more susceptible to develop injury, perhaps due the time of the WOD (little exercise intensity time).

The training load (AU) demonstrated higher values in EAA when compared with all the other athletes. One study evaluating the reliability of TRIMP in athletes through Firstbeat ${ }^{\circledR}$ showed this software to be a good predictor of training load [25]. CrossFit athletes training weekly about $2092 \mathrm{AU}$ [26]. In one international competition there is often 6 to 10 events, indicating that the intensity/volume of training is even higher in than competition amount, but there is many factors in competition that can interfere the performance (sound, climate, events in a row, etc).

EAA exposes higher EPOC than all of the other athletes. EPOC is correlated with HR in Firstbeat ${ }^{\oplus}$, so as higher the training intensity is (RPE), higher is the EPOC after the WOD for a longer time. The high EPOC value demonstrate a possible better adaptation after some days of appropriated rest, as cited in the study of Herbert et al. [27].

The present study researched physiological adaptation in a real time of a great CrossFit competition.

Training effect, EPOC and RPE demonstrated that EAA was less adapted when comparing with all the others (individual and team), and this can possibly have relationship with the limitation of the study. EAD seems to be the more adapted athlete (team) to the semi-final, through his physiological data (MHR, training load (AU), EPOC, $\mathrm{RPE}$ and training effect). It is important to emphasize that EAD was $4 \mathrm{x}$ winner Brazilian Championship (TCB), but he didn't classify to the finals, perhaps due his team.
Using this data, a coach staff may be better met the individual needs of athletes, competing individually or team sports. This method may increase the like hood of maintaining good performance without overtraining. Also, it is important for trainers and coaches to develop balanced WODs that do not exacerbate the volume and intensity in the same way.

\section{Limitation of the study}

This study is not without limitation: 1) the study analysed only 5 athletes due the complexity and difficult to contact the elite athletes in this competition organization level; 2) EAA had recent surgery, so it was expected that he wasn't in his best performance.

\section{Conflict of interest}

The authors have no conflicts of interest to disclose.

\section{Acknowledgment:}

We would like to thank André Berzoti for all the technological help.

\section{References}

1. Tibana RA, de Sousa NMF (2018) Are extreme conditioning programs effective and safe? A narrative review of high-intensity functional training methods research paradigms and findings. BMJ Open Sport Exerc Med [Crossref]

2. Barnes KR, Kilding AE (2015) Strategies to improve running economy. Sports Med 45 37-56. [Crossref]

3. Kilpatrick MW, Jung M, Little JP (2014) High-intensity interval training: A Review of Physiological and Psychological Responses. ACSM's Helath Fit J 18: 11-16.

4. Mate-Munoz JL, Lougedo JH, Barba M, Canuelo-Marquez AM, Guodemar-Perez J, et al. (2018) Cardiometabolic and Muscular Fatigue Responses to Different CrossFit(R) Workouts. J Sport Sci Med 17: 668-679. [Crossref]

5. Glassman G (2007) Understanding Crossfit. Crossfit J 1: 1-2

6. Maxwell Ruth K, Friesen CC (2017) Sports Nutrition Knowledge, Perceptions, Resources, and Advice Given by Certified CrossFit Trainers. Sports 5: 2-9. [Crossref]

7. Bellar D, Hatchett A, Judge LW, Breaux ME, Marcus L (2015) The relationship of aerobic capacity, anaerobic peak power and experience to performance in CrossFit exercise. Biol Sport 32: 315-320. [Crossref]

8. Perciavalle V, Marchetta NS, Giustiniani S, Borbone C, Perciavalle V, et al. (2016) Attentive processes, blood lactate and CrossFit(R). Phys Sport. 44: 403-406; [Crossref]

9. Tibana RA, Prestes J, Sousa N (2019) Time-Course of Changes in Physiological, Psychological and Performance markers Following a Functional-Fitness Competition. [Crossref]

10. Smith Sommer AJ, Starkoff SB, Devor ST (2013) Crossfit-based high intensity power training improves maximal aerobicfitness and body composition. University of wisconsin-LA Crosse.

11. Pata ET (2017) High intensity interval training for older adults. University of Saskatchewan.

12. Schubert MM, Palumbo EA (2019) Energy balance dynamics during short-term highintensity functional training. Appl Physiol Nutr Metab 44: 172-178. [Crossref]

13. Malinauskas R, Malinauskiene V (2018) The mediation effect of perceived social support and perceived stress on the relationship between emotional intelligence and psychological wellbeing in male athletes. J Hum Kinet 65: 291-303. [Crossref]

14. Meeusen R, De-Pauw K (2019) Overreaching and overtraining syndrome: Causes, prevention, and remedy. 2nd ed. Washington, DC, US: American Psychological Association.: APA handbooks in psychology. 147-157.

15. Borg GA V (1982) Psychophysical bases of perceived exertion. Med Sci Sports Exerc 14: 377-381. [Crossref]

16. Foster C, Florhaug JA, Franklin J, Gottschall L, Hrovatin LA, et al. (2001) A new approach to monitoring exercise training. J Strength Cond Res 15: 109-115. [Crossref]

17. Kraemer WJ, Adams K, Cafarelli E, Dudley GA, Dooly C, et al. (2002) American College of Sports Medicine position stand. Progression models in resistance training for healthy adults. Med Sci Sport Exerc 34: 364-80. [Crossref] 
18. Dexheimer JD, Schroeder ET, Sawyer BJ, Pettitt RW, Aguinaldo AL, et al. (2019) Physiological Performance Measures as Indicators of CrossFit ${ }^{\circledR}$ Performance. Sports 7: 13 .

19. Tibana R, de Sousa N, Cunha G, Prestes J, Fett C, Gabbett T, et al. Validity of Session Rating Perceived Exertion Method for Quantifying Internal Training Load during High-Intensity Functional Training. Sports 6: 68. [Crossref]

20. Shaw BS, Dullabh M, Forbes G, Brandkamp JL, Shaw I (2015) Analysis of physiological determinants during a single bout of crossfit. Int J Perform Anal Sport 15: 809-815.

21. Astorino TA, Martin BJ, Wong K, Schachtsiek L (2011) Effect of acute caffeine ingestion on EPOC after intense resistance training. J Sport Med Phys Fit 51: 11-7. [Crossref]

22. Williams S, Booton T, Watson M, Rowland D, Altini M (2017) Heart rate variability is a moderating factor in the workload-injury relationship of competitive crossfitTM athletes. J Sport Sci Med 16: 443-449. [Crossref]
23. Tibana RA, de Almeida LM, Frade de Sousa NM, Nascimento Dda C, Neto I V, et al. (2016) Two Consecutive Days of Crossfit Training Affects Pro and Anti-inflammatory Cytokines and Osteoprotegerin without Impairments in Muscle Power. Front Physiol 7: 260. [Crossref]

24. Ulmer JG, Tomkinson GR, Short S, Short M, et al. (2019) Test-retest reliability of TRIMP in collegiate ice hockey players. Biol Sport 36: 191-194. [Crossref]

25. Tibana RA, Sousa NMF de, Prestes J, Feito Y, Ferreira CE, et al. (2019) Monitoring Training Load, Well-Being, Heart Rate Variability, and Competitive Performance of a Functional-Fitness Female Athlete: A Case Study. Sports 7: 35. [Crossref]

26. Herbert P, Hayes LD, Sculthorpe NF, Grace FM (2017) HIIT produces increases in muscle power and free testosterone in male masters athletes. Endocr Connect 6: 430436. [Crossref]

Copyright: (C2020 Zecchin-Oliveira AM. This is an open-access article distributed under the terms of the Creative Commons Attribution License, which permits unrestricted use, distribution, and reproduction in any medium, provided the original author and source are credited. 